\title{
Optic Nerve Sheath Diameter Correlation with Elevated Intracranial Pressure Determined via Ultrasound
}

\author{
Kamran Munawar ${ }^{1}$, Muhammad Tariq Khan ${ }^{1}$, Syed Waqar Hussain ${ }^{2}$, Aayesha Qadeer ${ }^{1}$, \\ Zahid Siddique Shad ${ }^{1}$, Sheher Bano ${ }^{1}$, Azmat Abdullah ${ }^{1}$ \\ 1. Internal Medicine, Shifa International Hospital, Islamabad, PAK 2. Internal Medicine, Khan Research \\ Laboratories Hospital, Islamabad, PAK
}

$\square$ Corresponding author: Kamran Munawar,dr.kam18@gmail.com Disclosures can be found in Additional Information at the end of the article

\section{Abstract}

\section{Background}

The early detection of elevated intracranial pressure (ICP) can not only prevent mortality but also aid in more aggressive management. Brain computed tomography (CT) is a mainstay modality in detecting elevated ICP, but the feasibility of using brain CTs to detect elevated ICP in critically ill patients is limited, especially for patients who require high levels of inotropic support. The optic nerve sheath is a direct extension of the brain meninges. Therefore, the elevation of ICP is directly transmitted to the sheath. Measuring the optic nerve sheath diameter (ONSD) through ultrasound (US) is a bedside, noninvasive means to detect elevated ICP. The goal of this study was to assess the correlation of ONSD with elevated ICP as measured via US in an intensive care unit (ICU).

\section{Methods}

We conducted a six-month prospective, single-center, observational study of mass effect stroke patients aged 18 to 65 years who had a traumatic brain injury (TBI) and were admitted to the ICU. Patients with chronic hydrocephalus, extensive local orbit trauma, a pre-existing ocular disease affecting the optic nerve and/or orbital cavity, hyperthyroidism with exophthalmos, and facial trauma affecting the orbits and/or eyeballs were excluded. We measured the ONSD at the entry of optic nerve into the globe using two-dimensional (2D) US.

\section{Results}

One hundred patients were included in the study. Forty-nine patients had diffuse cerebral edema detected on CT scan correlating with increased ONSD notable via bedside US. The mean ONSD related to CT-detectable elevated ICP was $0.61 \mathrm{~cm}$. The sensitivity for the ONSD cut-off value of $\geqslant 5.8 \mathrm{~mm}$ was $94 \%$ (95\% confidence interval [CI], $84.05 \%$ to $98.79 \%$ ), and the specificity was $96.08 \%$ (95\% CI, $86.7 \%$ to $99.52 \%$ ).The positive predictive value was $92.08 \%$ (95\% CI, $86.28 \%$ to $98.96 \%$ ), and the negative predictive value was $94.23 \%$ (95\% CI, $84.47 \%$ to $98.00 \%$ ).

\section{Conclusion}

The greatest accuracy in ONSD was found with a cut-off of $>0.58 \mathrm{~cm}$ in patients with positive CT brain findings. Therefore, US can be used as an initial screening test when physicians suspect a patient has elevated ICP. 
Categories: Internal Medicine, Pulmonology

Keywords: onsd, icu, tbi, icp

\section{Introduction}

Elevated intracranial pressure (ICP) is a frequent problem in neurosurgical and neurological practice and may be due to inappropriate cerebrospinal fluid (CSF) circulation, intracranial mass lesions (including traumatic or spontaneous intracranial bleeding) or more diffuse intracranial pathological processes. Elevated ICP development may be acute or chronic, but it commonly results in a pressure gradient among compartments and a shift of brain structures causing changes in consciousness, bilateral ptosis, pupillary dilatation, and impaired up gaze. Elevated ICP can be assessed directly through the invasive measurement of intraventricular pressure [1]. However, elevated ICP can also be inferred by observing secondary effects associated with high ICP such as papilledema or dilatation of the cerebral ventricles. Lately, ocular ultrasound (US) for detecting dilatation of the optic nerve sheath diameter (ONSD) as a sign of elevated ICP has shown promising results. The optic nerve sheath is composed of dura mater and covers the optic nerve up to the back of the eye. Intracranial CSF is in direct connection with the rim of the subarachnoid space between the optic nerve sheath and the nerve itself. Therefore, any change in ICP will dynamically change the ONSD. When patients develop elevated ICP, ophthalmologists are usually consulted to assess the ONSD, as a clinical examination may be inadequate. Computed tomography (CT) and magnetic resonance imaging (MRI) are useful tools for detecting elevated ICP noninvasively. However, CT and MRI are not always indicated, especially in critically ill patients who require high levels of inotropic support or ventilator support. The use of bedside US has shown to be an equivalent if not improved means of measuring elevated ICP via assessment of the ONSD [2]. The aim of this study was to investigate the usefulness of using bedside US to measure OSND to determine if a patient has elevated ICP.

\section{Materials And Methods}

We conducted a six-month prospective, single-center, observational study in the intensive care unit (ICU) at Shifa International Hospital in Pakistan. The study included patients aged 18 to 65 years admitted to the ICU for stroke with mass effect causing traumatic brain injury (TBI). Patients with chronic hydrocephalus, extensive local orbit trauma, a pre-existing ocular disease affecting the optic nerve and/or orbital cavity and hyperthyroidism with exophthalmia, and facial trauma affecting the orbits and/or eyeballs were excluded from the study. The ONSD US findings were evaluated by trained fellows of critical care, and brain CT scan findings were masked to the US evaluators. The ONSD was measured via two-dimensional (2D) US scans at the entry of optic nerve into the globe. After sonography, a brain CT scan was conducted and reviewed by an expert radiologist and was compared with ONSD.

The hospital ethics committee approved the study protocol, and since the study was noninterventional, we did not require informed consent.

We followed a similar data collection to that described by Legrand et al. [3]. We collected patient age, Glasgow Coma Scale (GCS) score, and injury at the time of admission. We recorded the presence of signs showing elevated ICP on brain CT scan including massive intracerebral bleeding, subarachnoid hemorrhage with intraventricular extension, compression of the basal cistern, or midline shift $>5 \mathrm{~mm}$.

For an analytical description of quantitative variables, standard deviation and mean were used to assess the capacity for ONSD to predict brain CT results. We used a receiver operator characteristic (ROC) curve and its area under the curve to determine an optimal cut-off value for ONSD. Once the cut-off value was determined, we calculated the specificity, sensitivity, 


\section{Cureus}

negative predictive value (NPV), positive predictive value (PPV), negative likelihood ratio, and positive likelihood ratio. $\mathrm{P}<0.05$ was considered statistically significant. We used IBM SPSS Statistics for Windows, version 21.0 (IBM Corp., Armonk, NY) in applying the ROC analysis.

All CT scans included in the study were performed in the radiology department at Shifa International Hospital. All patient surgical and medical histories were masked to the radiologist, as were the nature of the TBI and GCS scores at the time of assessment. CT scans were performed before any intervention when needed. Brain CT scans were performed with a series of slices. ONSD was measured vertically $0.3 \mathrm{~cm}$ behind the optic nerve entering the globe, and at this point, the transverse diameter was obtained separately on both eyes. A mean ONSD was produced based on the right and left eye measurements.

\section{Results}

A total of 100 patients ( 53 men, 47 women) admitted to the ICU were evaluated in the study. The cutoff value for normal ONSD on 50 patients who had normal CT scans was $0.57 \mathrm{~cm}$. Fortynine patients with diffuse cerebral edema detected on CT scan also had increased ONSD on bedside ultrasonography. The average ONSD associated with elevated ICP detectable on CT was $0.61 \mathrm{~cm}$. The sensitivity for the ONSD cut-off value of $\geqslant 5.8 \mathrm{~mm}$ was $94 \%$ ( $95 \%$ confidence interval [CI], $84.05 \%$ to $98.79 \%$ ) and the specificity was $96.08 \%$ (95\% CI, $86.7 \%$ to $99.52 \%$ ); the PPV was $92.08 \%$ (95\% CI, $86.28 \%$ to $98.96 \%$ ), and the NPV was $94.23 \%$ (95\% CI, $84.47 \%$ to 98.00\%) (Table 1).

\begin{tabular}{lll} 
Positive if Greater Than or Equal To & Sensitivity & Specificity \\
\hline .00000 & 1.000 & 1.000 \\
.39500 & .959 & 1.000 \\
.41500 & .959 & .980 \\
.43000 & .918 & .980 \\
.44500 & .898 & .922 \\
.45500 & .898 & .902 \\
.46500 & .898 & .863 \\
.47500 & .898 & .765 \\
.48500 & .898 & .667 \\
.49500 & .898 & .529 \\
.50500 & .878 & .431 \\
.51500 & .878 & .314 \\
.52500 & .878 & .255 \\
.53500 & .878 & .176 \\
.54500 & .878 & .059 \\
.55500 & .878 & .118 \\
\hline
\end{tabular}




\begin{tabular}{|c|c|c|}
\hline .56500 & .857 & .020 \\
\hline .57500 & .837 & .020 \\
\hline .58500 & .714 & .020 \\
\hline .59500 & .694 & .020 \\
\hline .60500 & .673 & .020 \\
\hline .62000 & .612 & .020 \\
\hline .63500 & .531 & .020 \\
\hline .64500 & .490 & .020 \\
\hline .65500 & .449 & .020 \\
\hline .67000 & .429 & .020 \\
\hline .68500 & .347 & .020 \\
\hline .69500 & .286 & .000 \\
\hline .70500 & .265 & .000 \\
\hline .71500 & .143 & .000 \\
\hline .73000 & .122 & .000 \\
\hline .74500 & .082 & .000 \\
\hline .78000 & .020 & .000 \\
\hline 1.00000 & .000 & .000 \\
\hline .0000 & 1.000 & 1.000 \\
\hline 1.5000 & .061 & .980 \\
\hline 3.0000 & .000 & .000 \\
\hline
\end{tabular}

TABLE 1: Sensitivity and specificity of ultrasonography of optic nerve sheath diameter for diagnosis of elevated intracranial pressure

\section{Discussion}

Elevated ICP can result in many clinical and traumatic circumstances and is a life-threatening condition [4]. The "gold standard" methods for measuring elevated ICP can put critically ill patients at further risk due to the invasive nature of the intervention, and these gold standard approaches carry a notable risk for severe complications like hemorrhage, infection, and malfunction. Therefore, these approaches are not indicated in patients in critical condition [5].

The assessment of ONSD for ICP detection has been widely studied via CT scan, MRI, and US [6]. The use of US for ONSD measurement is a recent, novel bedside approach to assess for elevated ICP. Bedside US is noninvasive and can provide a binary, satisfactory diagnostic 
accuracy [7]. However, the studies supporting this claim were limited by various methodological flaws to reach definitive results.

An ONSD of $5.8 \mathrm{~mm}$ as a cut-off value provided acceptable specificity, sensitivity, PPV, and NPV for detecting elevated ICP. The likelihood of elevated ICP is adequately low when the ONSD is less than $5.0 \mathrm{~mm}$. A previous study reported the ONSD measurement cut-off value of $5.9 \mathrm{~mm}$ as an indicator of elevated ICP [8]. The lower PPV in that study, regardless of its higher specificity and sensitivity, could have been due to the smaller patient population with positive brain CT findings.

In the last few decades, numerous studies have revealed that ONSD can noninvasively reflect elevated ICP [9]. One such study even reported that an increase in ONSD occurs faster in the presence of elevated ICP than the signs visible via ophthalmoscope [10]. Girisgin et al. reported that the average ONSD in those patients with suspected elevated ICP was considerably higher than the average ONSD in healthy subjects [11]. Soldatos et al. reported that an ONSD $>5.7 \mathrm{~mm}$ measured non-invasively to determine ICP had 74\% sensitivity and 100\% specificity compared to results found via invasive intraparenchymal catheter [12]. Major et al. showed a correlation of brain CT scans with ONSD $>5 \mathrm{~mm}$ with a sensitivity of $86 \%$ and specificity of $100 \%$ [13]. A study of 156 children concluded that larger mean ONSD is associated with elevated ICP [14].

Best cut-off values for ONSD correlating to elevated ICP should be established and standardized for diverse subgroups categorized by age, gender, and condition. Optic nerve sonology use is limited due to its technical nature requiring specific expertise [12]. Sonography may not be possible in special situations such as patients with local area surgical wounds and or anatomical alterations due to trauma (e.g., head and facial trauma) [15].

Future studies shall be conducted in this regard to develop new and improved methods of ultrasonography in these critical situations, which provide an early, valuable and equivalent information if not above than CT scan brain.

\section{Conclusions}

The greatest accuracy of US screening of ONSD for suspected elevated ICP is with an ONSD of more than $0.58 \mathrm{~cm}$. Therefore, US ONSD screening can be a useful initial test for suspected elevated ICP, especially in patients for whom traditional CT scanning is not feasible.

\section{Additional Information Disclosures}

Human subjects: Consent was obtained by all participants in this study. Shifa International Hospital issued approval 1075-350-2018. The above-mentioned article has been approved by Shifa International Hospital Ethical Committee. Animal subjects: All authors have confirmed that this study did not involve animal subjects or tissue. Conflicts of interest: In compliance with the ICMJE uniform disclosure form, all authors declare the following: Payment/services info: All authors have declared that no financial support was received from any organization for the submitted work. Financial relationships: All authors have declared that they have no financial relationships at present or within the previous three years with any organizations that might have an interest in the submitted work. Other relationships: All authors have declared that there are no other relationships or activities that could appear to have influenced the submitted work.

\section{References}


1. Raboel PH, Bartek J Jr, Andresen M, Bellander BM, Romner B: Intracranial pressure monitoring: invasive versus non-invasive methods-a review. Crit Care Res Pract. 2012, 2012:950393. 10.1155/2012/950393

2. Zewdie A, Tesema H, Aklilu AT, et al.: Bedside ultrasound as a simple non-invasive method of assessing intracranial pressure in a limited resource setting. Afr J Emerg Med. 2016, 6:e1-e4. 10.1016/j.afjem.2016.06.002

3. Legrand A, Jeanjean P, Delanghe F, Peltier J, Lecat B, Dupont H: Estimation of optic nerve sheath diameter on an initial brain computed tomography scan can contribute prognostic information in traumatic brain injury patients. Crit Care. 2013, 17:R61. 10.1186/cc12589

4. Shen L, Wang Z, Su Z, et al.: Effects of intracranial pressure monitoring on mortality in patients with severe traumatic brain injury: a meta-analysis. PLoS One. 2016, 11:e0168901. 10.1371/journal.pone.0168901

5. Bekar A, Doğan S, Abas F, et al.: Risk factors and complications of intracranial pressure monitoring with a fiberoptic device. J Clin Neurosci. 2009, 16:236-40.

10.1016/j.jocn.2008.02.008

6. Kalantari H, Jaiswal R, Bruck I, Matari H, Ghobadi F, Weedon J, Hassen GW: Correlation of optic nerve sheath diameter measurements by computed tomography and magnetic resonance imaging. Am J Emerg Med. 2013, 31:1595-7. 10.1016/j.ajem.2013.07.028

7. Ohle R, McIsaac SM, Woo MY, Perry JJ: Sonography of the optic nerve sheath diameter for detection of raised intracranial pressure compared to computed tomography: a systematic review and meta-analysis. J Ultrasound Med. 2015, 34:1285-94. 10.7863/ultra.34.7.1285

8. Karakitsos D, Soldatos T, Gouliamos A, et al.: Transorbital sonographic monitoring of optic nerve diameter in patients with severe brain injury. Transplant Proc. 2006, 38:3700-6.

9. Hansen HC, Helmke K: Validation of the optic nerve sheath response to changing cerebrospinal fluid pressure: ultrasound findings during intrathecal infusion tests. J Neurosurg. 1997, 87:34-40. 10.3171/jns.1997.87.1.0034

10. Gangemi M, Cennamo G, Maiuri F, D'Andrea F: Echographic measurement of the optic nerve in patients with intracranial hypertension. Minim Invasive Neurosurg. 1987, 30:53-55.

10.1055/s-2008-1053656

11. Girisgin AS, Kalkan E, Kocak S, Cander B, Gul M, Semiz M: The role of optic nerve ultrasonography in the diagnosis of elevated intracranial pressure. Emerg Med J. 2007, 24:251-4. 10.1136/emj.2006.040931

12. Soldatos T, Karakitsos D, Chatzimichail K, Papathanasiou M, Gouliamos A, Karabinis A: Optic nerve sonography in the diagnostic evaluation of adult brain injury. Crit Care. 2008, 12:R67. 10.1186/cc6897

13. Major R, Girling S, Boyle A: Ultrasound measurement of optic nerve sheath diameter in patients with a clinical suspicion of raised intracranial pressure. Emerg Med J. 2011, 28:67981. 10.1136/emj.2009.087353

14. Malayeri AA, Bavarian S, Mehdizadeh M: Sonographic evaluation of optic nerve diameter in children with raised intracranial pressure. J Ultrasound Med. 2005, 24:143-7. 10.7863/jum.2005.24.2.143

15. Hassen GW, Bruck I, Donahue J, et al.: Accuracy of optic nerve sheath diameter measurement by emergency physicians using bedside ultrasound. J Emerg Med. 2015, 48:450-7. 10.1016/j.jemermed.2014.09.060 